\title{
ARE THE BAND WIDTH SYNTHESIS DELAY AND DELAY RATE OBSERVABLES USEFUL FOR VLBI IMAGING?
}

\author{
P. CHARLOT \\ Jet Propulsion Laboratory, California Institute of Technology, 4800 Oak \\ Grove Drive, Pasadena, CA 91109 \\ and \\ Observatoire de Paris, Central Bureau of IERS, 61 Avenue de l'Observatoi- \\ re, 75014 Paris, France
}

\begin{abstract}
The Band Width Synthesis (BWS) delay and delay rate observables measured by VLBI are used in astrometry to determine positions of extragalactic radio sources with a precision of $0.001^{\prime \prime}$. At this level of precision, extragalactic radio sources exhibit 'extended structures that induce significant effects in the measured BWS delays and delay rates. The question whether closure BWS delays and delay rates could supplement the amplitudes and closure phases for VLBI imaging is studied. Preliminary results for the radio source NRAO140 observed in July 1983 are presented.
\end{abstract}

\section{INTRODUCTION}

VLBI imaging is based on the hybrid-mapping algorithm developed by Readhead and Wilkinson (1978) and Cornwell and Wilkinson (1981). This algorithm uses the amplitude and closure phase observables measured by VLBI to infer brightness distributions of radio sources with angular resolutions at the level of $0.001^{\prime \prime}$. The other two observables measured by VLBI, the Band Width Synthesis (BWS) delay, defined by the group delay $\partial \phi / \partial \omega$ (where $\phi$ is the phase, and $\omega$ is the frequency), and the delay rate, defined by $\partial \phi / \partial t$ (where $t$ is the time), are presently used for astrometric and geodetic purposes only. However, they may also be useful for VLBI imaging, because they are sensitive to source structure effects (see Charlot 1990) at the current level of precision of astrometric VLBI $\left(0.001^{\prime \prime}\right)$. Structure information is revealed from these observables by forming closure delays and delay rates which depend on source structures only, similar to closure phases used in hybrid-mapping. Section 2 discusses the structure information contained in the closure BWS delays and delay rates, and the resulting gain in $u-v$ sampling if used to supplement the amplitudes and closure phases for VLBI imaging. Section 3 compares structure effects calculated from two models of the radio source NRAO140 observed in July 1983 with a six-station array. One of these models was fitted to the measured amplitudes and closure phases only, while the other was fitted to the measured values of all four VLBI observables (amplitudes, closure phases, closure delays, closure delay rates). 


\section{SOURCE STRUCTURE INFORMATION CONTAINED IN THE BWS} DELAY AND DELAY RATE OBSERVABLES

The BWS delay is the partial derivative of the phase with respect to frequency. When varying frequency, a single $u-v$ point moves across the corresponding $u-v$ track. Thus, the BWS delay contains information about the variations of the phase on both sides of a $u-v$ track. Using closure BWS delays to supplement closure phases for VLBI imaging would be similar to multifrequency synthesis imaging (see Conway these proceedings) with the difference that the first derivative of the phase replaces the individual phases measured in each frequency channel. The delay rate is the partial derivative of the phase with respect to time. When varying time, a single $u-v$ point moves along the corresponding $u-v$ track. Thus, the delay rate contains information about the variations of the phase along a $u-v$ track. Such information is useful to extrapolate the phase between the observed $u-v$ points when it varies rapidly or when there are gaps in the data.

Since they contain information about the variations of the phase in the $u-v$ plane, the BWS delay and delay rate observables may help in some cases to alleviate the problem of incomplete sampling of the $u-v$ plane if used for VLBI imaging. Such a statement is also supported by the ordinary sampling theory, as noted by Thomas (1980). This theory indicates that when measuring a function on the basis' of a uniform sample rate, the measurement of both the value and the derivative of the function at each sample point allows one to reduce the minimum sample rate to half the sample rate required for value-only sampling. In the case of aperture synthesis, the sample rate could be reduced from the Nyquist rate $R_{N}\left(R_{N}=2 \times\right.$ highest frequency) to $R_{N} / 2$ if accurate measurements of the closure BWS delay and delay rate are available. By using these two observables to supplement the amplitudes and closure phases for VLBI imaging, the effectiveness of a given set of $u-v$ tracks would be increased, and the $u-v$ sampling would be improved even if not adding more $u-v$ points.

\section{APPLICATION TO THE RADIO SOURCE NRAO140}

NRAO140 was observed as a calibrator at $5 \mathrm{GHz}$ during a VLBI experiment on radio stars with a six-station array in July 1983 (Lestrade et al. 1985). It was mapped by Charlot et al. (1989), hereafter CHL89, who also fitted a four-gaussian component model to the data (amplitudes and closure phases). The hybrid-CLEAN map of CHL89 is reproduced in Figure 1. Charlot (1990) studied variations of $B W S$ delay and delay rate structure effects calculated from the four-component model of CHL89, and noticed strong discrepancies between the measured and calculated closure delays and delay rates of NRAO140 when in low-visibility regions of the $u-v$ plane. In Figure 2, the dotted lines

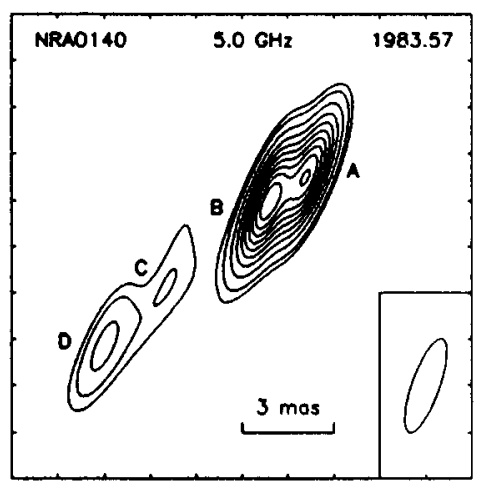

Fig. 1. Hybrid-CLEAN map of NRAO140 produced by CHL89. 


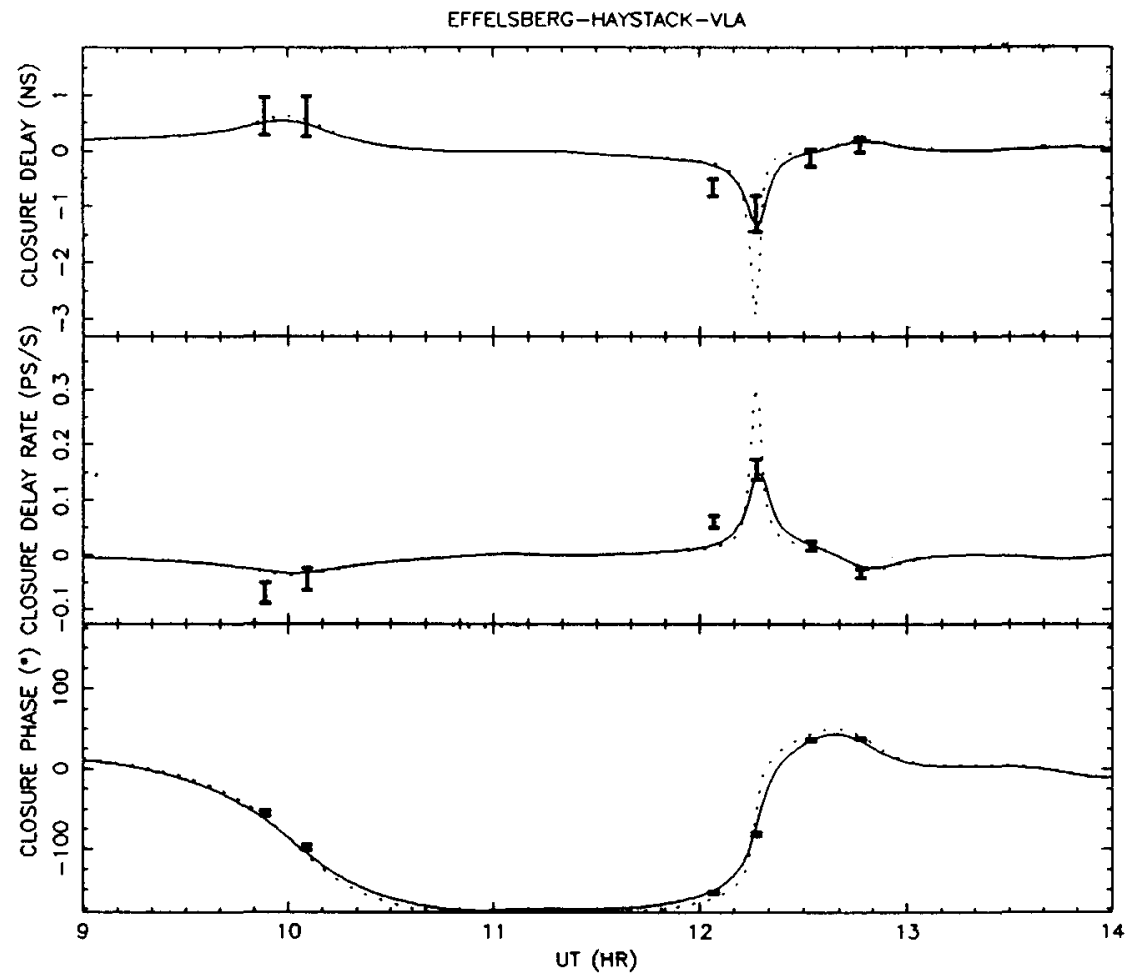

Fig. 2 Comparison of measured closure quantities (vertical bars) with closure quantities calculated from the model of CHL89 (dotted lines) and the model based on the four VLBI observables (continuous lines).

show variations of structure effects in closure delay, closure delay rate and closure phase for the intercontinental triangle Effelsberg-Haystack-VLA. It is noticeable that the intensities of the peaks in closure delay and closure delay rate at UT $12 \mathrm{~h} 15 \mathrm{~m}$ are at least twice as large as the actual closure delays and delay rates measured during the experiment.

By studying the sensitivity of BWS delay and delay rate observables to parameters of the model, we found that when varying the flux densities of the two main components (A,B) of NRAO140 by a few percents, the BWS delay and delay rate peak intensities in Figure 2 are changed by as much as several hundred percents, whereas the closure phases do not vary much. Thus, it is not possible to accurately predict these peak intensities from a model based only on closure phases, because they are less sensitive than the closure BWS delays and delay rates to flux density variations. In such a case, the only way to obtain a model that agrees well, not only with the measured amplitudes and closure phases, but also with the measured closure delays and delay rates, is to include these latter two observables in the least-squares adjustement of the model to the data. For this purpose, the program MODELFIT of the Caltech package was extended so 
that the measured values of all four VLBI observables (amplitude, closure phase, closure delay, closure delay rate) are accounted for in the adjustment. Using this modified version of MODELFIT, a new four-component model of NRAO140 was derived and variations of BWS delay, delay rate and phase structure effects were recalculated. These variations are plotted with the continuous lines in Figure 2. Although parameters of the new model, e.g. flux densities, differ by only a few percents from those of the model of CHL89, structure effects in BWS delay and delay rate at the epoch of the peaks are significantly different. The agreement between the measured and calculated closure delays and delay rates is much better when structure effects are derived from this new model, as shown in Figure 2. It is to be noted that the closure phase agreement is also slightly better in this case, which indicates that the new model of NRAO140 is closer to actual source structure than the model of CHL89. This new model is also useful to calculate BWS delay and delay rate structure corrections for astrometry and geodesy.

\section{CONCLUSION}

The BWS delay and delay rate observables measured by VLBI are sensitive to source structure effects. When summed around triangles, these observables reveal information about variations of the structure phase into unsampled regions of the $u-v$ plane, which may be useful in some cases to alleviate the problem of incomplete $u-v$ sampling in VLBI imaging. In the case of NRAO140, closure delays and delay rates have been used together with amplitudes and closure phases to fit a model of the structure consistent with the measured values of these four observables. The model derived with this method is improved with regard to the one of CHL89, based on amplitudes and closure phases only. It also shows that the parametrization of BWS delay and delay rate structure effects in astrometry is possible. Further investigations should be carried out to study whether closure $\mathrm{BWS}$ delays and delay rates can be included in the hybrid-mapping algorithm.

\section{ACKNOWLEDGEMENTS}

The author is grateful to J.-F. Lestrade for useful discussions. The research described here was carried out by the Jet Propulsion Laboratory, California Institute of Technology, under a contract with NASA, while P. C. was supported in part by a Lavoisier Fellowship of the Ministère des Affaires Etrangères, France.

\section{REFERENCES}

Charlot, P. 1990, A. J., 99, 1309.

Charlot, P., Hough, D. H., Lestrade, J.-F. 1989, Astr. Ap., 211, 261.

Cornwell, T. J., Wilkinson, P. N. 1981, M.N.R.A.S., 196, 1067.

Lestrade, J.-F., Mutel, R. L., Preston, R. A., Phillips, R. B. 1985, in Radio Stars, ed R. M. Hjellming and D. M. Gibson, Reidel, Dordrecht, p. 275.

Readhead, A. C. S., Wilkinson, P. N. 1978, Ap. J., 223, 25.

Thomas, J. B. 1980, NASA-JPL Publ. 80-84. 
Roger Linfield: If you sample the phases (or closure phases) frequently, the delay rate (actually a scaled phase rate) will not provide any additional constraints on source structure.

Patrick Charlot: What I have just said for the closure delays is also valid for the closure delay rates. If you sample the phases more frequently, you get as much as (or more) information than if using the delay rates-but, if not the case, the closure delay rates are useful to supplement the information contained in the closure phases.

John Conway: As you noted, your method has many parallels with the MFS technique. Have you considered using explicitly the amplitude and closure phase in each frequency channel (i.e. doing MFS mapping). This scheme must contain more information than using the closure delay. Patrick Charlot: I have not tried yet to do multi-frequency synthesis mapping. If you use explicitly the amplitudes and closure phases in each frequency channel, you do get more information than if using the closure delays, but it is a different approach. The approach I propose is intermediate between standard mapping and MFS mapping. 